\title{
Características do apoio social oferecido a idosos de área rural assistida pelo PSF
}

\author{
Characteristics of social support offered \\ rural elderly persons assisted by PSF
}

José Leonel Gonçalves Pinto 1

Adriana Carla de Oliveira Garcia 1

Silvia Cristina Mangini Bocchi 2

Maria Antonieta B. L. Carvalhaes 2

\footnotetext{
1 Secretaria Municipal da Saúde de Taquarituba Rua Quintino Bocaiuva 365, Centro, 18740-000, Taquarituba SP. jlpto2003@yahoo.com.br

2 Departamento

de Enfermagem da

Faculdade de Medicina de Botucatu, Unesp.
}

Abstract The present study is an epidemiological work of the transversal type, with the objective of describing characteristics of social support and identifying associations between sociodemographic variables and categories of social support, in the rural population of Bairro dos Aleixos, in the municipality of Taquarituba - São Paulo State - Brazil. To measure social support, the Medical Outcomes Study (MOS) scale was utilized, translated to Portuguese and validated in our environment. The population was characterized predominantly by white women between 60 and 69 years of age, married and/or living as concubines, of low incomes and residing in multigenerational domiciles. Elevated mean support scores were identified, indicating a favorable situation among the elderly persons studied. In average terms, the category of support with the worst score was "positive social interaction". Bivariate analysis demonstrated a positive association between the affective support score and emotional support with the number of persons in the domicile and greater frequency of high social-interaction scores ( 3 rd tercile) in elderly men and those with a greater degree of formal education. The study also showed that lower social-support scores were more frequent in women, illiterates, widowers or single men, and elderly persons with income between one and two times the minimum salary.

Key words Elderly, Social support, Health
Resumo Trata-se de um trabalho epidemiológico do tipo transversal, com o objetivo de descrever as características do apoio social e identificar associações entre as variáveis sociodemográficas e categorias de suporte social, em população rural do Bairro dos Aleixos, no município de Taquarituba - SP. Para medir o apoio social, utilizou-se da escala Medical Outcomes Study (MOS), traduzida para o português e validada em nosso meio. A população caracterizou-se por mulheres, predominantemente da raça branca, entre 60 a 69 anos, casadas e/ou vivendo em concubinatos, de baixa renda e residindo em domicílios multigeracionais. Escores de apoio médios elevados foram identificados, indicando situação favorável entre os idosos estudados. Em termos médios, a categoria de apoio com pior escore foi "interação social positiva". A análise bivariada demonstrou uma diferença significativa entre escore de apoio afetivo e de apoio emocional com número de pessoas no domicílio e maior freqüência de escores altos de interação social (3o tercil) em idosos do sexo masculino e naqueles com maior grau de escolaridade. O estudo apontou ainda que escores de apoio social menores foram mais freqüentes em mulheres, analfabetos, viúvos ou solteiros, idosos com renda entre um e dois salários mínimos. Palavras-chave Idoso, Apoio social, Saúde 


\section{Introdução}

A partir dos anos 80, o envelhecimento populacional tornou-se um fenômeno que atinge grande parte do mundo, tanto em países desenvolvidos quanto em países em desenvolvimento.

A população de 60 anos de idade ou mais, no Brasil, aumentou em quase quatro milhões de pessoas, fruto do crescimento vegetativo e do aumento gradual da esperança média de vida. Trata-se, certamente, de um conjunto bastante elevado de pessoas, com tendência de crescimento nos próximos anos. O segmento que, no período intercensitário, mais cresceu foi aquele das pessoas de 75 anos ou mais ${ }^{1}$.

O prolongamento do tempo de vida das pessoas tem suscitado inúmeros questionamentos sobre os condicionantes das condições de saúde dos idosos na sociedade atual. A impossibilidade de explicar integralmente diferenças na distribuição de enfermidades físicas e mentais pelos contrastes socioeconômicos e biológicos presentes na população tem motivado pesquisadores a estudar o papel de fatores psicossociais.

Existe uma literatura substancial que aponta a relação entre saúde dos idosos e relacionamentos sociais2, 3. A maioria dos autores ressalta que relações sociais satisfatórias parecem promover melhores condições de saúde², mas os mecanismos pelos quais este efeito é exercido ainda não são totalmente conhecidos. Sugere-se que a ajuda recebida e a ajuda oferecida contribuem para um senso de controle pessoal e isso tem uma influência positiva no bem-estar psicológico 4 . $\mathrm{O}$ apoio social poderia tanto proteger os indivíduos dos efeitos patogênicos de eventos estressantes - efeito amortecedor ${ }^{5}$ quanto afetar direta e positivamente a saúde das pessoas ao fornecer recursos (ajuda econômica, material, informações), melhor acesso ao cuidado de saúde e regulação de hábitos como consumo de álcool e tabaco.

Estudos relacionaram altos escores de satisfação com a vida e melhor estado de saúde (segundo auto-avaliação) em idosos com maior freqüência de contatos com irmãos 6 e com familiares e amigos 7,8 .

Apoio social é um conceito em construção que envolve ao mesmo tempo a estrutura da rede de relacionamentos sociais e a adequação de sua função, especialmente o grau de satisfação da pessoa com o apoio social de que usufrui.

A diferença entre rede social e suporte social é que a primeira pode ser definida como um conjunto de relacionamentos de um indivíduo ou de elos entre um conjunto de pessoas, enquanto a segunda enfoca a qualidade das interações e como estas são avaliadas pelo indivíduo receptor ${ }^{9}$.

De acordo com Thoits 10 e Due et al.11, o apoio social é composto de estrutura e função, com aspectos e fenômenos distintos. A estrutura das relações sociais refere-se à organização do vínculo entre pessoas e pode ser descrita sob diferentes aspectos, tais como: número de relações ou papéis sociais que uma pessoa tem, freqüência de contatos com vários membros de uma rede, entre outros. É composta pela rede de relações formais e informais. As relações formais são mantidas devido à posição e papéis desempenhados na sociedade, como: médico, dentista, advogado, professor, entre outras profissões; ao passo que as relações informais são tidas como de maior importância pessoal e afetiva do que as relações mais especializadas e formais. São compostas por todos os indivíduos (família, amigos, vizinhos, colegas de trabalho, comunidade, entre outros) e pela ligação entre indivíduos com quem se tem uma relação familiar próxima ou com envolvimento afetivo. "Medir" a estrutura de rede social permite avaliar o grau em que um indivíduo está socialmente conectado com os outros, seu nível de isolamento ou de integração social.

Com vistas a operacionalizar o conceito de apoio social e encontrar instrumentos para sua medida, foram propostas cinco categorias funcionais de apoio social $12,13,14$ :

1) Apoio material: refere-se ao apoio direcionado ao auxílio das pessoas em caso de necessidade. Reflete o acesso dos indivíduos aos serviços práticos e recursos materiais, em que estão incluídos, por exemplo, a ajuda em dinheiro ou empréstimo de utensílios, em caso de necessidade emergencial.

2) Apoio afetivo: envolve expressões de amor e afeição.

3) Apoio emocional: refere-se à empatia, carinho, amor, confiança, estima, afeto, escuta e interesse.

4) Apoio de informação: refere-se a informações que podem ser usadas para lidar com problemas. É medido por meio do acesso dos indivíduos a aconselhamentos, sugestões, orientações e informações.

5) Interação social positiva: é a disponibilidade de pessoas com quem se divertir e relaxar.

Este estudo teve como objetivos: descrever a situação do apoio social a idosos de área rural 
assistida pelo Programa Saúde da Família (PSF); identificar associações entre fatores socioeconômicos e demográficos e cada categoria de apoio social acima apresentada; identificar subgrupos de idosos mais expostos à inadequação do apoio social.

Acredita-se que esses dados serão úteis para chamar a atenção de equipes do PSF para a importância do apoio social como condicionante de saúde, e para a possibilidade de uma atuação que identifique o perfil do idoso mais vulnerável a deficiências no apoio social, reforçando e/ou auxiliando a estruturação de eficientes redes de apoio de idosos em sua área de atuação, com prováveis reflexos positivos sobre suas condições de vida e saúde.

\section{Redes de apoio social em idosos}

A revisão da literatura tem demonstrado que do mesmo modo como as redes sociais de apoio influenciam as condições de saúde e mortalidade da população em geral, a presença de apoio social tem sido fortemente associada com desfechos positivos, também, para os idosos.

$\mathrm{O}$ efeito protetor das relações sociais sobre a mortalidade tem sido amplamente reconhecido por meio de estudos epidemiológicos longitudinais realizados com idosos em vários continentes e países, tais como: Estados Unidos15, 16, Europa17, 18 19, 20, Ásia21 e a África ${ }^{22}$. Redes sociais diversificadas associaram-se, significantemente, a algumas medidas de saúde (capacidade funcional, incontinência urinária, autoavaliação de saúde e problemas visuais) para idosos 23 .

Além das investigações relacionando a presença de redes sociais com redução de mortalidade, a importância deste fator em idosos tem sido evidenciada principalmente no campo da saúde mental. Tais trabalhos mostram que sintomas depressivos estão inversamente associados com o tamanho das redes sociais 24 ; mostram também que existe uma relação direta entre grau de incapacidade e sintomas depressivos, e que o suporte social alivia o efeito da incapacidade dos idosos em depressão, quando a rede de apoio é avaliada positivamente por eles 25 . Outros estudos relacionam altos escores de satisfação com a vida, melhor estado de saúde na auto-avaliação de idosos - importantes preditores de mortalidade - com freqüência de contatos com irmãos ${ }^{6}$ e com freqüência de contatos com familiares e amigos7, 8. Tem-se tam- bém observado na população de idosos a associação positiva entre redes sociais formadas por grande número de amigos e escores de estado de ânimo 26 e auto-estima 27.

Há estudos demonstrando os efeitos da integração social em comportamentos alimentares. Autores como Learner \& Kivett 28 verificaram que os idosos que estavam satisfeitos com a freqüência de visitas recebidas por parentes e amigos registraram menos problemas com suas dietas. Ademais, Krondl et al. ${ }^{29}$ observaram que os idosos mais ativos física e emocionalmente tinham dietas alimentares mais diversificadas e adequadas. Finalmente, McIntosh et al. 30 constataram que amplas redes de amigos têm conseqüências positivas no apetite e na ingestão adequada de nutrientes e, além disso, a magnitude dos efeitos negativos do estresse financeiro sobre o apetite foi reduzida pelo relacionamento com amigos, pelo estado conjugal e pela presença de companhia.

A preferência dos idosos por diferentes tipos de apoio tem se tornado o foco da atenção de pesquisas gerontológicas. Pinquart \& Sörensen 8 observaram, numa população americana e canadense, que os idosos preferem apoio informal e misto (formal/informal) para necessidades de cuidados em curto prazo e preferem assistência mais formal para necessidades de cuidados de longo prazo. Um estudo realizado na China, contexto cultural e econômico totalmente diferente do estudo anterior, evidencia resultados guardando certa semelhança, enquanto os idosos chineses esperam que os programas de pensão do Estado resolvam uma boa parte das suas necessidades financeiras, a família permanece preferencialmente como fonte de apoio social e emocional, tanto nas áreas urbanas quanto rurais ${ }^{31}$.

\section{Método}

Trata-se de um estudo epidemiológico do tipo transversal, realizado no município de Taquarituba, estado de São Paulo, com a população idosa rural do Bairro dos Aleixos. O município possui 21.982 habitantes, dos quais 9,34\% são idosos. A principal atividade econômica na região é a agropecuária. $\mathrm{Na}$ área de atuação do PSF, onde este estudo foi realizado, residiam 60 idosos. Destes foram entrevistados 52 , pois oito indivíduos se recusaram a participar da pesquisa.

Os dados foram coletados pelos pesquisadores, no próprio domicílio do idoso, em horá- 
rio e local adequados para salvaguardar a privacidade de suas informações.

O instrumento de coleta de dados foi composto por duas partes: parte I, com questões destinadas à identificação da pessoa, bem como a levantar dados socioeconômicos e demográficos; parte II, com questões relativas ao apoio material, apoio afetivo, apoio emocional, apoio de informação e interação social positiva. Utilizou-se o Medical Outcomes StudyMOS14, traduzido para o português e validado por estudo que o aplicou em população do Rio de Janeiro, obtendo bons resultados de consistência interna, estabilidade e validade de constructo $^{32}$.

As perguntas referentes a cada dimensão de apoio social foram agrupadas em cinco escores independentes. Esses índices foram padronizados para que variassem entre 20 e 100, independentemente do número de perguntas que compunham cada dimensão. Tal padronização foi realizada por meio da razão entre as somas dos pontos obtidos no conjunto das perguntas de cada dimensão e o valor máximo de pontos possíveis de serem obtidos, de acordo com o número de perguntas de cada dimensão. O resultado dessa razão foi multiplicado por 100 . Por exemplo, se o idoso respondeu "sempre", "quase sempre" e "nunca" às três perguntas relativas ao apoio afetivo, obteve um total de 10 pontos $(5+4+1)$. Este total foi dividido por 15 pontos, que representa o máximo que se poderia atingir caso o idoso respondesse "sempre" nas três perguntas $(5+5+5)$. O resultado obtido foi multiplicado por 10032 .

Para as análises, os escores foram divididos em tercis. É importante salientar que, quanto maior o escore alcançado, maior o nível de apoio social. Assim, o 1o tercil ficou constituído pelos escores mais baixos, o 2 o tercil pelos níveis intermediários e o 3 o é o tercil composto pelos escores mais altos ${ }^{32}$.

Com interesse em identificar idosos mais expostos à inadequação do apoio social, foram feitas análises para identificar a associação entre alguns fatores: gênero, estado civil, renda, número de pessoas no domicílio, com tercis de apoio, aplicando-se o teste não-paramétrico de Kruskal-Wallis (KW) ao nível de significância de 5\%.

A análise dos dados foi realizada por meio do programa estatístico Epi Info 2002 e organizada em forma de tabelas.

\section{Resultados}

Na tabela 1 são apresentadas as características socioeconômicas e demográficas da população estudada: $57,7 \%$ estavam na faixa etária de 60 a 69 anos e 7,8\% acima de 80 anos; houve um ligeiro predomínio $(51,9 \%)$ do sexo feminino; de "não-alfabetizados" (51,9\%), de casados e/ ou vivendo em concubinato $(53,8 \%)$ e brancos $(78,8 \%)$. Quanto à renda familiar, mais de dois terços viviam com até dois salários mínimos mensais. Houve um predomínio de idosos $(57,7 \%)$ vivendo em domicílios com três a nove indivíduos. Caracteriza-se assim uma população de baixo nível socioeconômico.

Na tabela 2, apresentam-se as médias e respectivos desvios padrão, as medianas e os valores que limitam os tercis dos escores de apoio social. Valores médios próximos de 80 e medianas em torno de 90 foram obtidos para as várias categorias de apoio; apenas para o escore de interação social positiva a média foi de 75 pontos. Os limites dos tercis dos escores de apoio também foram semelhantes para as várias categorias de apoio, exceto para o escore “interação social positiva”.

Na tabela 3, verificou-se que não houve diferença significativa com faixa etária, sexo, escolaridade, renda e número de pessoas na família. Apenas para número de pessoas houve tendência de escores mais baixos em famílias com uma ou duas pessoas em comparação com famílias mais numerosas.

Na tabela 4, evidenciou-se que o número de pessoas residentes no domicílio apresentou diferença significativa em relação ao apoio afetivo com o p-valor de 0,00. Quanto maior o número de pessoas no domicílio, maior a freqüência de idosos no tercil superior de apoio afetivo.

Observamos também que, entre idosos casados e com maior renda, houve maior freqüência de indivíduos no terceiro tercil do escore de apoio afetivo, mas sem alcançar significância estatística.

Na tabela 5, entre as variáveis sociodemográficas pesquisadas, somente o número de pessoas residentes no domicílio apresentou diferença significativa com escore de apoio emocional através do p-valor de 0,03 .

Como pode ser observada na tabela 6 , nenhuma variável apresentou diferença significativa com escore de apoio de informação.

Observando-se a tabela 7 , verifica-se que houve diferença significativa entre sexo e escolaridade com tercis de interação social positiva, 


\begin{tabular}{|c|c|c|}
\hline \multicolumn{3}{|c|}{$\begin{array}{l}\text { Tabela } 1 \\
\text { Freqüências relativas das variáveis sociodemográficas da população rural de idosos do Bairro } \\
\text { dos Aleixos no município de Taquarituba-SP, } 2003 .\end{array}$} \\
\hline Variáveis & $\mathbf{N}$ & $\%$ \\
\hline \multicolumn{3}{|l|}{ Idade (anos) } \\
\hline 60 a 69 & 30 & 57,7 \\
\hline 70 a 79 & 18 & 34,5 \\
\hline$\geq 80$ & 4 & 7,8 \\
\hline \multicolumn{3}{|l|}{ Sexo } \\
\hline Feminino & 27 & 51,9 \\
\hline Masculino & 25 & 48,1 \\
\hline \multicolumn{3}{|l|}{ Escolaridade } \\
\hline Não-alfabetizado & 27 & 51,9 \\
\hline Somente alfabetizado até a $3 a$ série do ensino fundamental & 17 & 32,7 \\
\hline $\mathrm{Da} 4 \underline{\mathrm{a}}$ a $8 \underline{\mathrm{a}}$ série do ensino fundamental & 8 & 15,4 \\
\hline \multicolumn{3}{|l|}{ Situação conjugal } \\
\hline Solteiro & 1 & 1,9 \\
\hline Viúvo & 23 & 44,2 \\
\hline Casado ou vivendo em concubinato & 28 & 53,8 \\
\hline \multicolumn{3}{|l|}{ Raça } \\
\hline Branca & 41 & 78,8 \\
\hline Negra & 7 & 13,5 \\
\hline Parda & 4 & 7,7 \\
\hline \multicolumn{3}{|l|}{ Renda familiar (em salários mínimos) } \\
\hline 1 a 2 & 19 & 36,5 \\
\hline$>2$ a 3 & 19 & 36,5 \\
\hline$>3$ & 14 & 28,0 \\
\hline \multicolumn{3}{|l|}{ Pessoas residentes no domicílio } \\
\hline 1 & 5 & 9,6 \\
\hline 2 & 17 & 32,7 \\
\hline 3 a 9 & 30 & 57,7 \\
\hline
\end{tabular}

\section{Tabela 2}

Médias, desvios padrão, medianas e limites dos tercis dos escores relativos as categorias de apoio social da população de idosos do Bairro dos Aleixos no município de Taquarituba-SP, 2003.

\begin{tabular}{lccccc}
\hline Dimensões de apoio social & NO de itens & Média & Desvio padrão & Mediana & Valores limitantes dos tercis \\
\hline Material & 4 & 80,76 & 22,10 & 90,00 & $25 \mathrm{a} \leq 70 ; \geq 75 \mathrm{a} \leq 95 ; 100$ \\
Afetivo & 3 & 81,79 & 23,20 & 86,70 & $20 \mathrm{a} \leq 73,3 ; \geq 80 \mathrm{a} \leq 93,3 ; 100$ \\
Emocional & 4 & 81,07 & 23,50 & 90,00 & $25 \mathrm{a} \leq 75 ; \geq 80 \mathrm{a} 95 ; 100$ \\
Informação & 4 & 80,58 & 23,44 & 90,00 & $20 \mathrm{a} \leq 75 ; \geq 80 \mathrm{a} 95 ; 100$ \\
Interação social positiva & 4 & 75,19 & 29,22 & 90,00 & $20 \mathrm{a} \leq 55 ; \geq 70 \mathrm{a} 95 ; 100$ \\
\hline
\end{tabular}




\section{Tabela 3}

Valor e p-valor do teste Kruskal-Wallis para as associações entre as características sociodemográficas da população rural de idosos e os valores limitantes dos tercis de apoio material dos mesmos no município de Taquarituba-SP, 2003.

\begin{tabular}{|c|c|c|c|c|c|c|c|c|c|}
\hline \multirow{3}{*}{$\begin{array}{l}\text { Variáveis } \\
\text { sociodemográficas }\end{array}$} & \multicolumn{6}{|c|}{ Tercis de apoio material } & \multirow{2}{*}{\multicolumn{2}{|c|}{$\begin{array}{l}\text { Total } \\
\mathrm{N}=52\end{array}$}} & \multirow{3}{*}{$\begin{array}{l}\text { Teste KW } \\
\text { p-valor }\end{array}$} \\
\hline & \multicolumn{2}{|c|}{$\mathrm{T}_{1}$} & \multicolumn{2}{|c|}{$\mathrm{T}_{2}$} & \multicolumn{2}{|c|}{$\mathrm{T}_{3}$} & & & \\
\hline & $\mathrm{n}$ & $\%$ & $\mathrm{n}$ & $\%$ & $\mathrm{n}$ & $\%$ & $\mathrm{n}$ & $\%$ & \\
\hline \multicolumn{10}{|l|}{ Faixa etária } \\
\hline 60 a 69 & 12 & 40,0 & 7 & 23,3 & 11 & 36,7 & 30 & 100,0 & 1,56 \\
\hline 70 a 79 & 4 & 22,2 & 6 & 33,3 & 8 & 44,4 & 18 & 100,0 & 0,45 \\
\hline$\geq 80$ & 1 & 25,0 & 1 & 25,0 & 2 & 50,0 & 4 & 100,0 & (n.s) \\
\hline \multicolumn{10}{|l|}{ Sexo } \\
\hline Feminino & 7 & 25,9 & 8 & 29,6 & 12 & 44,4 & 27 & 100,0 & 1,15 \\
\hline Masculino & 10 & 40,0 & 6 & 24,0 & 9 & 36,0 & 25 & 100,0 & $\begin{array}{l}0,56 \\
\text { (n.s) }\end{array}$ \\
\hline \multicolumn{10}{|l|}{ Situação conjugal } \\
\hline Solteiro & 0 & 0,0 & 0 & 0,0 & 1 & 100,0 & 1 & 100,0 & 1,37 \\
\hline Viúvo & 6 & 26,1 & 7 & 30,4 & 10 & 43,5 & 23 & 100,0 & 0,50 \\
\hline Casado ou concubinato & 11 & 39,3 & 7 & 25,0 & 10 & 35,7 & 28 & 100,0 & (n.s.) \\
\hline \multicolumn{10}{|l|}{ Escolaridade } \\
\hline Não-alfabetizado & 8 & 29,6 & 9 & 33,3 & 10 & 37,0 & 27 & 100,0 & 1,05 \\
\hline $\begin{array}{l}\text { Somente alfabetizado } \\
\text { até a } 3 \text { a série do ensino } \\
\text { fundamental }\end{array}$ & 8 & 47,1 & 3 & 17,6 & 6 & 35,3 & 17 & 100,0 & 0,59 \\
\hline $\begin{array}{l}\mathrm{Da} 4 \mathrm{a} \text { a } 8 \text { a série do } \\
\text { ensino fundamental }\end{array}$ & 1 & 12,5 & 2 & 25,0 & 5 & 62,5 & 8 & 100,0 & (n.s) \\
\hline \multicolumn{10}{|l|}{ Renda familiar } \\
\hline 1 a 2 & 8 & 42,1 & 5 & 26,3 & 6 & 31,6 & 19 & 100,0 & 1,74 \\
\hline$>2$ a 3 & 6 & 31,6 & 5 & 26,3 & 8 & 42,1 & 19 & 100,0 & 0,41 \\
\hline$>3$ & 3 & 21,4 & 4 & 28,6 & 7 & 50,0 & 14 & 100,0 & (n.s) \\
\hline \multicolumn{10}{|l|}{$\begin{array}{l}\text { Pessoas residentes } \\
\text { no domicílio }\end{array}$} \\
\hline 1 & 2 & 40,0 & 2 & 40,0 & 1 & 20,0 & 5 & 100,0 & 4,9 \\
\hline 2 & 8 & 47,1 & 5 & 29,4 & 4 & 23,5 & 17 & 100,0 & 0,08 \\
\hline 6 a 9 & 7 & 23,3 & 7 & 23,3 & 16 & 53,3 & 30 & 100,0 & (n.s) \\
\hline
\end{tabular}

com os respectivos p-valor, 0,03 e 0,04. Entre os homens e entre idosos com mais alta escolaridade, houve maior freqüência do terceiro tercil.

\section{Discussão}

Este estudo apontou que a população de idosos da área rural do Bairro dos Aleixos, em Taquarituba-SP, atendida pelo PSF, em termos médios apresenta apoio social adequado, em suas diferentes dimensões. De certa forma, estes foram resultados diferentes do esperado, considerando as precárias condições de vida vigentes na população estudada. Chamou a atenção o fato de que os resultados foram semelhantes para as diversas dimensões de apoio avaliadas, reforçando a constatação de adequação do apoio disponível.

Apenas para o escore de interação social positiva, a média foi menor. Assim, podemos afirmar que a população de idosos do Bairro dos Aleixos percebe, entre as dimensões de apoio social, que a sua dificuldade maior é participar de atividades sociais de entretenimento, capazes de proporcionar momentos de diversão e descontração. Demonstrou-se haver diferenças significantes entre os tercis dessa dimensão de apoio e as variáveis sexo e escolaridade. Assim, os homens e os indivíduos com maior escolari- 


\begin{tabular}{|c|c|c|c|c|c|c|c|c|c|}
\hline $\begin{array}{l}\text { Tabela } 4 \\
\text { Valor e p-valor do teste Kru } \\
\text { da população rural de idoso } \\
\text { no município de Taquaritu }\end{array}$ & $\begin{array}{l}1-\mathrm{Wa} \\
\text { os va } \\
\mathrm{PP}, 20\end{array}$ & $\begin{array}{l}\text { para } \\
\text { res lim }\end{array}$ & $\begin{array}{l}\text { soci } \\
\text { tes d }\end{array}$ & $\begin{array}{l}\text { ões entr } \\
\text { s tercis }\end{array}$ & $\begin{array}{l}\text { car: } \\
\text { poio }\end{array}$ & $\begin{array}{l}\text { rística } \\
\text { tivo d }\end{array}$ & $\begin{array}{l}\text { ciode } \\
\text { nesm }\end{array}$ & ográfic & \\
\hline \multirow{3}{*}{$\begin{array}{l}\text { Variáveis } \\
\text { sociodemográficas }\end{array}$} & \multicolumn{6}{|c|}{ Tercis de apoio afetivo } & \multirow{2}{*}{\multicolumn{2}{|c|}{$\begin{array}{r}\text { Total } \\
\mathrm{N}=51\end{array}$}} & \multirow{3}{*}{$\begin{array}{c}\text { Teste KW } \\
\text { p-valor }\end{array}$} \\
\hline & \multicolumn{2}{|c|}{$\mathrm{T}_{1}$} & \multicolumn{2}{|c|}{$\mathrm{T}_{2}$} & \multicolumn{2}{|c|}{$\mathrm{T}_{3}$} & & & \\
\hline & $\mathrm{n}$ & $\%$ & $\mathrm{n}$ & $\%$ & $\mathrm{n}$ & $\%$ & $\mathrm{n}$ & $\%$ & \\
\hline \multicolumn{10}{|l|}{ Faixa etária } \\
\hline 60 a 69 & 10 & 33,3 & 11 & 36,7 & 9 & 30,0 & 30 & 100,0 & 2,91 \\
\hline 70 a 79 & 2 & 11,8 & 6 & 35,3 & 9 & 52,9 & 17 & 100,0 & 0,23 \\
\hline$\geq 80$ & 1 & 25,0 & 1 & 25,0 & 2 & 50,0 & 4 & 100,0 & (n.s.) \\
\hline \multicolumn{10}{|l|}{ Sexo } \\
\hline Feminino & 6 & 23,1 & 9 & 34,6 & 11 & 42,3 & 26 & 100,0 & 0,25 \\
\hline Masculino & 7 & 28,0 & 9 & 36,0 & 9 & 36,0 & 25 & 100,0 & $\begin{array}{l}0,88 \\
\text { (n.s.) }\end{array}$ \\
\hline \multicolumn{10}{|l|}{ Situação conjugal } \\
\hline Solteiro & 0 & 0,0 & 1 & 100,0 & 0 & 0,0 & 1 & 100,0 & 3,38 \\
\hline Viúvo & 8 & 36,4 & 8 & 36,4 & 6 & 27,3 & 22 & 100,0 & 0,18 \\
\hline Casado ou concubinato & 5 & 17,9 & 9 & 32,1 & 14 & 50,0 & 28 & 100,0 & (n.s.) \\
\hline \multicolumn{10}{|l|}{ Escolaridade } \\
\hline Não-alfabetizado & 6 & 23,1 & 11 & 42,3 & 9 & 34,6 & 26 & 100,0 & 1,40 \\
\hline $\begin{array}{l}\text { Somente alfabetizado } \\
\text { até a } 3 a \text { a série do ensino } \\
\text { fundamental }\end{array}$ & 6 & 35,3 & 5 & 29,4 & 6 & 35,3 & 17 & 100,0 & 0,49 \\
\hline $\begin{array}{l}\text { Da } 4 \text { a a } 8 \underline{a} \text { série do } \\
\text { ensino fundamental }\end{array}$ & 1 & 12,5 & 2 & 25,0 & 5 & 62,5 & 8 & 100,0 & (n.s.) \\
\hline \multicolumn{10}{|l|}{ Renda familiar } \\
\hline$\leq 2$ & 7 & 36,8 & 7 & 36,8 & 5 & 26,3 & 19 & 100,0 & 3,86 \\
\hline$>2$ a 3 & 5 & 27,8 & 5 & 27,8 & 8 & 44,4 & 18 & 100,0 & 0,14 \\
\hline$>3$ & 1 & 7,1 & 6 & 42,9 & 7 & 50,0 & 14 & 100,0 & (n.s.) \\
\hline \multicolumn{10}{|l|}{$\begin{array}{l}\text { Pessoas residentes } \\
\text { no domicílio }\end{array}$} \\
\hline 1 & 4 & 80,0 & 1 & 20,0 & 0 & 0,0 & 5 & 100,0 & 11,09 \\
\hline 2 & 6 & 35,3 & 6 & 35,3 & 5 & 29,4 & 17 & 100,0 & 0,00 \\
\hline 3 a 9 & 3 & 10,3 & 11 & 37,9 & 15 & 51,7 & 29 & 100,0 & (s.) \\
\hline
\end{tabular}

dade desfrutavam de melhor interação social. Estes resultados apontam, por um lado, a maior vulnerabilidade das mulheres no que se refere a esta importante dimensão do apoio social. Por outro lado, os idosos com menor escolaridade, e possivelmente menor nível socioeconômico, têm menos acesso a interações destinadas à diversão e ao lazer.

Dados brasileiros apontam que os homens idosos são, proporcionalmente, mais alfabetizados do que as mulheres nesta mesma idade, favorecidos pelas características da sociedade e das políticas de educação prevalentes nas décadas de 1930 e 1940, quando o acesso de mulheres à escola era muito restrito ${ }^{1}$. A maior susce- tibilidade da mulher idosa ao isolamento social, em termos principalmente de interações ligadas à diversão e ao lazer, parece ser reflexo da divisão sexual do trabalho, produto do modelo de família nuclear considerado hegemônico em referencial e em ideal de ordenação da vida doméstica para a grande maioria da população. Nesse tipo de organização familiar, as atividades da mulher estão circunscritas ao domicílio, vinculadas à sua organização e à preservação da saúde e da educação dos filhos, o que a torna mais reclusa em relação ao contato social quando comparada ao homem. São fatos culturais que podem contribuir para a adoção de uma postura de isolamento social no está- 


\section{Tabela 5}

Valor e p-valor do teste Kruskal-Wallis para as associações entre as características sociodemográficas da população rural de idosos e os valores limitantes dos tercis de apoio emocional dos mesmos no município de Taquarituba-SP, 2003.

\begin{tabular}{|c|c|c|c|c|c|c|c|c|c|}
\hline \multirow{3}{*}{$\begin{array}{l}\text { Variáveis } \\
\text { sociodemográficas }\end{array}$} & \multicolumn{6}{|c|}{ Tercis de apoio emocional } & \multirow{2}{*}{\multicolumn{2}{|c|}{$\begin{array}{l}\text { Total } \\
\mathrm{N}=51\end{array}$}} & \multirow{3}{*}{$\begin{array}{l}\text { Teste KW } \\
\text { p-valor }\end{array}$} \\
\hline & \multicolumn{2}{|c|}{$\mathrm{T}_{1}$} & \multicolumn{2}{|c|}{$\mathrm{T}_{2}$} & \multicolumn{2}{|c|}{$\mathrm{T}_{3}$} & & & \\
\hline & $\mathrm{n}$ & $\%$ & $\mathrm{n}$ & $\%$ & $\mathrm{n}$ & $\%$ & $\mathrm{n}$ & $\%$ & \\
\hline \multicolumn{10}{|l|}{ Faixa etária } \\
\hline 60 a 69 & 9 & 30,0 & 8 & 26,7 & 13 & 43,3 & 30 & 100,0 & 0,28 \\
\hline 70 a 79 & 5 & 29,4 & 5 & 29,4 & 7 & 41,2 & 17 & 100,0 & 0,87 \\
\hline$\geq 80$ & 2 & 50,0 & 0 & 0,0 & 2 & 50,0 & 4 & 100,0 & (n.s.) \\
\hline \multicolumn{10}{|l|}{ Sexo } \\
\hline Feminino & 8 & 30,8 & 7 & 26,9 & 11 & 42,3 & 26 & 100,0 & 0,05 \\
\hline Masculino & 8 & 32,0 & 6 & 24,0 & 11 & 44,0 & 25 & 100,0 & $\begin{array}{l}0,97 \\
\text { (n.s.) }\end{array}$ \\
\hline \multicolumn{10}{|l|}{ Situação conjugal } \\
\hline Solteiro & 0 & 0,0 & 0 & 0,0 & 1 & 100,0 & 1 & 100,0 & 0,17 \\
\hline Viúvo & 8 & 36,4 & 6 & 27,3 & 8 & 36,4 & 22 & 100,0 & 0,92 \\
\hline Casado ou concubinato & 8 & 28,6 & 7 & 25,0 & 13 & 46,4 & 28 & 100,0 & (n.s.) \\
\hline \multicolumn{10}{|l|}{ Escolaridade } \\
\hline Não-alfabetizado & 7 & 26,9 & 8 & 30,8 & 11 & 42,3 & 26 & 100,0 & 0,53 \\
\hline $\begin{array}{l}\text { Somente alfabetizado } \\
\text { até a } 3 \text { a série do ensino } \\
\text { fundamental }\end{array}$ & 7 & 41,2 & 3 & 17,6 & 7 & 41,2 & 17 & 100,0 & 0,77 \\
\hline $\begin{array}{l}\mathrm{Da} 4 \mathrm{a} \text { a } 8 \text { a série do } \\
\text { ensino fundamental }\end{array}$ & 2 & 25,0 & 2 & 25,0 & 4 & 50,0 & 8 & 100,0 & (n.s.) \\
\hline \multicolumn{10}{|l|}{ Renda familiar } \\
\hline$\leq 2$ & 7 & 36,8 & 4 & 21,1 & 8 & 42,1 & 19 & 100,0 & 2,60 \\
\hline$>2$ a 3 & 8 & 44,4 & 4 & 22,2 & 6 & 33,3 & 18 & 100,0 & 0,27 \\
\hline$>3$ & 1 & 7,1 & 5 & 35,7 & 8 & 57,1 & 14 & 100,0 & (n.s.) \\
\hline \multicolumn{10}{|l|}{$\begin{array}{l}\text { Pessoas residentes } \\
\text { no domicílio }\end{array}$} \\
\hline 1 & 3 & 60,0 & 0 & 0,0 & 2 & 40,0 & 5 & 100,0 & 6,48 \\
\hline 2 & 8 & 47,1 & 4 & 23,5 & 5 & 29,4 & 17 & 100,0 & 0,03 \\
\hline 3 a 9 & 5 & 17,2 & 9 & 31,0 & 15 & 51,7 & 29 & 100,0 & (s.) \\
\hline
\end{tabular}

gio tardio da vida e afetar negativamente a saúde, principalmente a saúde mental.

Há estudos mostrando que sintomas depressivos estão inversamente associados ao tamanho das redes sociais 24 , que altos escores de satisfação com a vida ocorrem em idosos com mais contatos com amigos e familiares 7 . A literatura enfatiza ainda haver associação entre contato social, apoio e longevidade, ou seja, a maioria dos idosos que visita seus amigos e familiares provavelmente viverá mais tempo do que aqueles que raramente têm contatos ${ }^{33}$.

Quanto ao apoio material, cujo escore reflete o acesso dos indivíduos a serviços práticos e a recursos materiais como, por exemplo, aju- da em dinheiro ou empréstimo de utensílios em caso de necessidade emergência 12,13 , 14, era esperado que idosos vivendo em famílias com maior renda obtivessem melhores escores. No entanto, não foi encontrada esta associação. É possível que a pequena diversidade socioeconômica presente na população estudada - todos eram indivíduos de baixa renda, de área rural e assistidos pelo PSF - tenha impedido essa identificação. Entretanto, observaram-se tendências (não estaticamente significativas) de que quanto maior o número de pessoas que residiam no domicílio, maior o escore de apoio material, sinalizando que residências multigeracionais, em áreas rurais, possam ser uma es- 


\begin{tabular}{|c|c|c|c|c|c|c|c|c|c|}
\hline \multicolumn{10}{|l|}{$\begin{array}{l}\text { Tabela } 6 \\
\text { Valor e p-valor do teste Kru } \\
\text { da população rural de idoso } \\
\text { no município de Taquaritul }\end{array}$} \\
\hline \multirow{3}{*}{$\begin{array}{l}\text { Variáveis } \\
\text { sociodemográficas }\end{array}$} & \multicolumn{6}{|c|}{ Tercis de apoio de informação } & \multirow{2}{*}{\multicolumn{2}{|c|}{$\begin{array}{l}\text { Total } \\
\mathrm{N}=51\end{array}$}} & \multirow{3}{*}{$\begin{array}{c}\text { Teste KW } \\
\text { p-valor }\end{array}$} \\
\hline & \multicolumn{2}{|c|}{$\mathrm{T}_{1}$} & \multicolumn{2}{|c|}{$\mathrm{T}_{2}$} & \multicolumn{2}{|c|}{$\mathrm{T}_{3}$} & & & \\
\hline & $\mathrm{n}$ & $\%$ & $\mathrm{n}$ & $\%$ & $\mathrm{n}$ & $\%$ & $\mathrm{n}$ & $\%$ & \\
\hline \multicolumn{10}{|l|}{ Faixa etária } \\
\hline 60 a 69 & 8 & 26,7 & 10 & 33,3 & 12 & 40,0 & 30 & 100,0 & 1,2 \\
\hline 70 a 79 & 6 & 35,3 & 5 & 29,4 & 6 & 35,3 & 17 & 100,0 & 0,53 \\
\hline$\geq 80$ & 2 & 50,0 & 0 & 0,0 & 2 & 50,0 & 4 & 100,0 & (n.s.) \\
\hline \multicolumn{10}{|l|}{ Sexo } \\
\hline Feminino & 10 & 38,5 & 8 & 30,8 & 8 & 30,8 & 26 & 100,0 & 1,81 \\
\hline Masculino & 6 & 24,0 & 7 & 28,0 & 12 & 48,0 & 25 & 100,0 & $\begin{array}{c}0,40 \\
\text { (n.s.) }\end{array}$ \\
\hline \multicolumn{10}{|l|}{ Situação conjugal } \\
\hline Solteiro & 0 & 0,0 & 0 & 0,0 & 1 & 100,0 & 1 & 100,0 & 0,20 \\
\hline Viúvo & 8 & 36,4 & 7 & 31,8 & 7 & 31,8 & 22 & 100,0 & 0,90 \\
\hline Casado ou concubinato & 8 & 28,6 & 8 & 28,6 & 12 & 42,9 & 28 & 100,0 & (n.s.) \\
\hline \multicolumn{10}{|l|}{ Escolaridade } \\
\hline Não-alfabetizado & 11 & 42,3 & 6 & 23,1 & 9 & 34,6 & 26 & 100,0 & 3,24 \\
\hline $\begin{array}{l}\text { Somente alfabetizado } \\
\text { até a } 3 a \text { a série do ensino } \\
\text { fundamental }\end{array}$ & 4 & 23,5 & 6 & 35,3 & 7 & 41,2 & 17 & 100,0 & 0,20 \\
\hline $\begin{array}{l}\text { Da } 4 \text { a a } 8 \underline{a} \text { série do } \\
\text { ensino fundamental }\end{array}$ & 1 & 12,5 & 3 & 37,5 & 4 & 50,0 & 8 & 100,0 & (n.s.) \\
\hline \multicolumn{10}{|l|}{ Renda familiar } \\
\hline 1 a 2 & 7 & 36,8 & 7 & 36,8 & 5 & 26,3 & 19 & 100,0 & 3,19 \\
\hline$>2$ a 3 & 7 & 38,9 & 4 & 22,2 & 7 & 38,9 & 18 & 100,0 & 0,20 \\
\hline$>3$ & 2 & 14,3 & 4 & 28,6 & 8 & 57,1 & 14 & 100,0 & (n.s.) \\
\hline \multicolumn{10}{|l|}{$\begin{array}{l}\text { Pessoas residentes } \\
\text { no domicílio }\end{array}$} \\
\hline 1 & 2 & 40,0 & 1 & 20,0 & 2 & 40,0 & 5 & 100,0 & 2,37 \\
\hline 2 & 7 & 41,2 & 7 & 41,2 & 3 & 17,6 & 17 & 100,0 & 0,18 \\
\hline 3 a 9 & 7 & 24,1 & 7 & 24,1 & 15 & 51,7 & 29 & 100,0 & (n.s.) \\
\hline
\end{tabular}

tratégia eficiente de obtenção de apoio material. Em futuros estudos, com amostra maior, esta hipótese deverá ser investigada.

Entretanto, há um estudo que levanta a hipótese de que os domicílios multigeracionais, antes de serem uma opção cultural ou humanitária, podem ser um arranjo de sobrevivência capaz de colocar o idoso numa situação de isolamento e dependência maior do que se ele estivesse morando só, principalmente quando passar a necessitar de freqüentes cuidados médicos e assistenciais ${ }^{34}$. Como já apontado, os dados do presente estudo não apontam nesta direção.

Outro resultado que indica os domicílios multigeracionais como mais capazes de prover apoio ao idoso diz respeito aos resultados das análises sobre apoio emocional. Os manifestos de empatia, carinho, amor, confiança, estima, afeto, escuta e interesse, percebidos pelas pessoas, são os aspectos incluídos na categoria apoio emocional12, 13, 14. Este estudo demonstrou diferença significativa entre as variáveis número de pessoas residentes no domicílio e escore de apoio emocional. Este foi maior em idosos vivendo em domicílios onde residiam de três a nove pessoas, quando comparados com aqueles vivendo sozinhos ou apenas com mais uma pessoa.

Os dados por nós obtidos também sugerem, mas sem alcançar significância estatística, 


\section{Tabela 7}

Valor e p-valor do teste Kruskal-Wallis para as associações entre as características sociodemográficas da população rural de idosos e os valores limitantes dos tercis de interação social positiva dos mesmos no município de Taquarituba-SP, 2003.

\begin{tabular}{|c|c|c|c|c|c|c|c|c|c|}
\hline \multirow{3}{*}{$\begin{array}{l}\text { Variáveis } \\
\text { sociodemográficas }\end{array}$} & \multicolumn{6}{|c|}{ Tercis de interação social positiva } & \multirow{2}{*}{\multicolumn{2}{|c|}{$\begin{array}{c}\text { Total } \\
\mathrm{N}=51\end{array}$}} & \multirow{3}{*}{$\begin{array}{c}\text { Teste KW } \\
\text { p-valor }\end{array}$} \\
\hline & \multicolumn{2}{|c|}{$\mathrm{T}_{1}$} & \multicolumn{2}{|c|}{$\mathrm{T}_{2}$} & \multicolumn{2}{|c|}{$\mathrm{T}_{3}$} & & & \\
\hline & $\mathrm{n}$ & $\%$ & $\mathrm{n}$ & $\%$ & $\mathrm{n}$ & $\%$ & $\mathrm{n}$ & $\%$ & \\
\hline \multicolumn{10}{|l|}{ Faixa etária } \\
\hline 60 a 69 & 8 & 26,7 & 10 & 33,3 & 12 & 40,0 & 30 & 100,0 & 3,06 \\
\hline 70 a 79 & 7 & 41,2 & 3 & 17,6 & 7 & 41,2 & 17 & 100,0 & 0,21 \\
\hline$\geq 80$ & 2 & 11,8 & 50,0 & 0,0 & 2 & 50,0 & 4 & 100,0 & (n.s.) \\
\hline \multicolumn{10}{|l|}{ Sexo } \\
\hline Feminino & 12 & 46,2 & 3 & 11,5 & 11 & 42,3 & 26 & 100,0 & 6,55 \\
\hline Masculino & 5 & 20,0 & 10 & 40,0 & 10 & 40,0 & 25 & 100,0 & $\begin{array}{c}0,03 \\
\text { (s.) }\end{array}$ \\
\hline \multicolumn{10}{|l|}{ Situação conjugal } \\
\hline Solteiro & 0 & 0,0 & 1 & 100,0 & 0 & 0,0 & 1 & 100,0 & 1,13 \\
\hline Viúvo & 8 & 36,4 & 6 & 27,3 & 8 & 36,4 & 22 & 100,0 & 0,56 \\
\hline Casado ou concubinato & 9 & 32,1 & 6 & 21,4 & 13 & 46,4 & 28 & 100,0 & (n.s.) \\
\hline \multicolumn{10}{|l|}{ Escolaridade } \\
\hline Não-alfabetizado & 10 & 38,5 & 9 & 34,6 & 7 & 26,9 & 26 & 100,0 & 6,26 \\
\hline $\begin{array}{l}\text { Somente alfabetizado } \\
\text { até a } 3 \text { a série do ensino } \\
\text { fundamental }\end{array}$ & 5 & 29,4 & 4 & 23,5 & 8 & 47,1 & 17 & 100,0 & 0,04 \\
\hline $\begin{array}{l}\text { Da } 4 \text { a a } 8 \text { a série do } \\
\text { ensino fundamental }\end{array}$ & 2 & 25,0 & 0 & 0,0 & 6 & 75,0 & 8 & 100,0 & (s.) \\
\hline \multicolumn{10}{|l|}{ Renda familiar } \\
\hline$\leq 2$ & 8 & 42,1 & 5 & 26,3 & 6 & 31,6 & 19 & 100,0 & 1,27 \\
\hline$>2$ a 3 & 5 & 27,8 & 5 & 27,8 & 8 & 44,4 & 18 & 100,0 & 0,53 \\
\hline$>3$ & 4 & 28,6 & 3 & 21,4 & 7 & 50,0 & 14 & 100,0 & (n.s.) \\
\hline \multicolumn{10}{|l|}{$\begin{array}{l}\text { Pessoas residentes } \\
\text { no domicílio }\end{array}$} \\
\hline 1 & 2 & 40,0 & 1 & 20,0 & 2 & 40,0 & 5 & 100,0 & 5,11 \\
\hline 2 & 9 & 52,9 & 5 & 29,4 & 3 & 17,6 & 17 & 100,0 & 0,08 \\
\hline 3 a 9 & 6 & 20,7 & 7 & 24,1 & 16 & 55,2 & 29 & 100,0 & (n.s.) \\
\hline
\end{tabular}

que os idosos com maior escolaridade e renda familiar, bem como os casados/concubinos, dispunham de apoio afetivo mais satisfatório quando comparados aos solteiros e/ou viúvos e aos de níveis de escolaridade e renda familiar menores.

Em estudo realizado por Ramos et al.34, o suporte emocional foi a necessidade substancial menos alcançada pelos idosos não-casados e pelos idosos sem filhos, representando, segundo os autores, uma ameaça para a qualidade de vida dos mesmos.

Outro estudo, realizado na cidade Guadalajara, México, evidenciou que as redes sociais dos idosos eram compostas de 7,5 integrantes em média, principalmente mulheres. Eram redes multigeracionais, proporcionando, em sua maioria, apoio emocional. As autoras acrescentam que as mulheres e os casados e viúvos tinham redes maiores e recebiam mais apoio de natureza emocional que os homens e os solteiros ${ }^{35}$.

A dimensão do apoio social relativa ao acesso à informação, que pode ser necessária e utilizada para se lidar com problemas, também apresentou escore médio elevado na população estudada. Este escore foi medido por meio de perguntas sobre o acesso dos indivíduos a aconselhamentos, a sugestões, a orientações e a informações 12, 13, 14 e, tratando-se de população pobre e rural, também eram esperados resulta- 
dos menos favoráveis. Apesar de não ter ocorrido diferença significativa entre as variáveis sociodemográficas e os tercis de apoio de informação, os dados sugerem que o apoio de informação pode também ser maior entre os idosos do sexo masculino e para aqueles com níveis de escolaridade e renda familiar maiores.

Finalizando, os resultados obtidos permitiram identificar, entre a população rural do Bairro dos Aleixos, município de Taquarituba, o perfil do idoso com mais chance de inadequação do suporte social: mulheres, analfabetos, viúvos/solteiros, idosos com rendimento entre um e dois salários mínimos e idosos vivendo com menor número de pessoas.

As características do apoio social, sem dúvida, devem ser consideradas por aqueles que organizam e prestam assistência à saúde de idosos, particularmente equipes do PSF. Na área onde este estudo foi realizado, este não parece ser um aspecto muito adverso.

\section{Colaboradores}

JLG Gonçalves, ACO Garcia, SCM Bocchi e MABL Carvalhaes participaram igualmente de todas as etapas de elaboração do artigo.

\section{Agradecimento}

Aos idosos participantes deste estudo, bem como ao Ministério da Saúde, em parceria com a Faculdade de Medicina de Botucatu-Unesp, por meio do Projeto Reforsus/ BID, nossos agradecimentos por nos terem concedido a oportunidade de realizar esta pesquisa, como requisito de conclusão do curso de Especialização em Saúde da Família.

\section{Conclusões}

O processo investigatório, norteado pelos objetivos, permitiu-nos chegar às seguintes conclusões: os idosos avaliados apresentaram escores médios de apoio social elevados e satisfatórios; entre as dimensões de apoio social, a interação social positiva foi a que obteve menor média; houve diferença significativa entre número de pessoas no domicílio e maior escore de apoio afetivo; o escore de apoio emocional foi maior nos domicílios onde residem mais de três pessoas; escores mais altos de interação social ocorreram em idosos do sexo masculino e naqueles com maior grau de escolaridade.

Com relação ao perfil do idoso com maior probabilidade de apresentar alguma inadequação de apoio social, predominam: mulheres, analfabetos, viúvos ou solteiros, idosos com renda entre um e dois salários mínimos.

\section{Referências}

1. Brasil. Ministério do Planejamento, Orçamento e Gestão. Instituto Brasileiro de Geografia e Estatística. Perfil dos idosos responsáveis pelos domicílios no Brasil. Rio de Janeiro: IBGE; 2002.

2. House JS, Robbins C, Metzner HL The association of social relationships and activities with mortality: prospective evidence from the Tecumseh Community Health Study. Am J Epidemiol 1998; 116(1):123-40.

3. Cockerham W. This aging society. New Jersey: Prentice Hall; 1991.

4. Ramos MP. Apoio social e saúde entre os idosos. Sociologias 2002; 4(7):156-75.

5. Berkman LF, Syme SL. Social networks, host resistance, and mortality: a nine-year follow-up study of Alameda County residents. Am J Epidemiol 1999; 109(2):186-204.

6. McCamish-Svensson C, Samuelsson G, Hagberg B, Svensson T, Dehlin O. Social relationships and health as predictors of life satisfaction in advanced old age: results from a Swedish longitudinal study. Int J Aging Hum Dev 1999; 48(4):301-24.

7. Thompson MG, Heller K. Facets of support related to well-being quantitative social isolation and perceived family support in a sample of elderly women. Psychol Aging 1990; 5(4)535-44. 
8. Pinquart M, Sörensen S. Older adults' preferences for informal, formal, and mixed support for future care needs: a comparison of Germany and the United States. Int J Aging Hum Dev 2002; 54(4):291-314.

9. Roth P. Family social support. In: Bomar PJ, organizador. Nurses and family health promotion: concepts, assessment, and interventions. Baltimore: Willians \& Wilkins; 1989. p. 90-102.

10. Thoits PA. Stress, coping, and social support processes: where are we? What next? J Health Soc Behav 1995; Spec No:53-79.

11. Due P, Holstein B, Lund R, Modvig J, Avlund K. Social relations: network, support and relational strain. Soc Sci Med 1999; 48(5):661-73.

12. Bowling A. Measuring social networks and social support. In: __. Measuring health: a review of quality of life measurements scales. Buckingham: Open University Press; 1997. p. 91-109.

13. Ostergren PO, Hanson BS, Isacsson SO, Tejler L. Social network, social support and acute chest complaints among young and middle-aged patients in an emergency department: a case-control study. Soc Sci Med 1991; 33(3):257-67.

14. Sherboune CD, Sterwart AL. The MOS social support survey. Soc Sci Med 1991; 32(6):705-14.

15. Davis MA, Moritz DJ, Neuhaus JM, Barclay JD, Gee L. Living arrangements, changes in living arrangements, and survival among community dwelling older adults. Am J Public Health 1997; 87(3):371-7.

16. Yasuda N, Zimmerman SI, Hawkes W, Fredman L, Hebel JR, Magaziner J. Relation of social network characteristics to 5-year mortality among young-old versus old-old white women in an urban community. Am J Epidemiol 1997; 145(6):516-23.

17. Shye D, Mullooly JP, Freeborn DK, Pope CR. Gender differences in the relationship between social network support and mortality: a longitudinal study of an elderly cohort. Soc Sci Med 1995; 41(7):935-47.

18. Penninx BW, van Tilburg T, Kriegsman DM, Deeg DJ, Boeke AJ, van Eijk JT. Effects of social support and personal coping resources on mortality in older age: the longitudinal aging study Amsterdam. Am J Epidemiol 1997; 146(6):510-9.

19. Fuhrer R, Stansfeld SA, Chemali J, Shipley MJ. Gender, social relations and mental health: prospective findings from an occupational cohort (Whitehall II study). Soc Sci Med 1999; 48(1):77-87.

20. Lund R, Modvig J, Due P, Holstein BE. Stability and change in structural social relations as predictor of mortality among elderly women and men. Eur J Epidemiol 2000; 16(2):1087-97.
21. Ceria CD, Masaki KH, Rodriguez BL, Chen R, Yano $\mathrm{K}$, Curb JD. The relationship of psychosocial factors to total mortality among older Japanese-American men: the Honolulu heart program. J Am Geriatr Soc 2001; 49(6):725-31.

22. Rahman MO. Age and gender variation in the impact of household structure on elderly mortality. Int J Epidemiol 1999; 28(3):485-91.

23. Litwin H. The provision of informal support by elderly people residing in assisted living facilities. Gerontologist 1998; 38(2):239-46.

24. Palinkas AL, Wingard DL, Barret-Cannor E. The biocultural context of social networks and depression among the elderly. Soc Sci Med 1990; 30(4):442-7.

25. Wallsten SM, Tweed DL, Blazer DG, George LK. Disability and depressive symptoms in the elderly: the effects of instrumental support and its subjective appraisal. Int J Aging Hum Dev 1999; 48(2):145-59.

26. Litwin $\mathrm{H}$. Social network type and morale in old age. Gerontologist 2001; 41(4):516-24

27. Lee GR, Shehan CL Social relations and the self-esteem of older persons. Res Aging 1989; 11(4):427-42.

28. Learner RM, Kivett VR. Discriminators of perceived dietary adequacy among the rural elderly. J Am Diet Assoc 1981; 78(4):330-7.

29. Krondl M, Lau D, Yurkiw MA, Coleman PH. Food use and perceived food meanings of the elderly. J Am Diet Assoc 1982; 80(6):523-9.

30. McIntosh WA, Shifflett PA, Picou JS. Social support, stressful events, strain, dietary intake, and the elderly. Med Care 1989; 27(2):140-53.

31. Pei X, Pillai VK. Old age support in China: the role of the state and the family. Int J Aging Hum Dev Development 1999; 49(3):197-212.

32. Andrade CR. Associação entre apoio social e freqüência relatada de auto-exame das mamas no estudo pró-saúde [dissertação]. Rio de Janeiro: Escola Nacional de Saúde, Fundação Oswaldo Cruz; 2001.

33. Wash F. A família em estágio tardio da vida. In: Carter B, McGoldrick M, organizadores. As mudanças no ciclo de vida familiar: uma estrutura para a terapia familiar. Porto Alegre: Artes Médicas; 1995. p. 269-87

34. Ramos LR, Rosa TEC, Oliveira ZM, Medina MCG, Santos FRG. Perfil do idoso em área metropolitana na região Sudeste do Brasil: resultados de inquérito domiciliar. Rev Saúde Pública 1993; 27(2):87-94.

35. Robles L, Curiel GR, García LMC, Coles LC, Medrano MSG, González MS. Redes y apoyo social en ancianos enfermos de escasos recursos en Guadalajara, México. Cad Saúde Pública; 16(2):557-60.

Artigo apresentado em 1ㅇ/04/2005

Aprovado em 3/05/2005

Versão final apresentada em 20/07/2005 